in vivo $32: 403-408(2018)$

doi:10.21873/invivo. 11253

\title{
Tear Film Osmolarity in Subjects with Acute Allergic Rhinoconjunctivitis
}

\author{
EIRINI NITODA ${ }^{1}$, ANASTASIOS LAVARIS ${ }^{1}$, KONSTANTINOS LAIOS $^{1}$, \\ SOPHIA ANDROUDI ${ }^{2}$, CHRIS D. KALOGEROPOULOS ${ }^{3}$, MICHAEL TSATSOS ${ }^{4}$, \\ CHRISTOS DAMASKOS ${ }^{5,6}$, NIKOLAOS GARMPIS ${ }^{5,6}$ and MARILITA M. MOSCHOS ${ }^{1}$ \\ ${ }^{1}$ First Department of Ophthalmology, General Hospital of Athens G. Gennimatas, \\ Medical School, National and Kapodistrian University of Athens, Athens, Greece; \\ ${ }^{2}$ Department of Ophthalmology, University of Thessaly, Larissa, Greece; \\ ${ }^{3}$ Department of Ophtalmology, Medical School, University of Ioannina, Ioannina, Greece; \\ ${ }^{4}$ Department of Ophthalmology, University Hospital Southampton, Southampton, U.K.; \\ ${ }^{5}$ Second Department of Propedeutic Surgery, Laiko General Hospital, Medical School, \\ National and Kapodistrian University of Athens, Athens, Greece; \\ ${ }^{6}$ N.S. Christeas Laboratory of Experimental Surgery and Surgical Research, \\ Medical School, National and Kapodistrian University of Athens, Athens, Greece
}

\begin{abstract}
Background/Aim: Acute allergic rhinoconjuctivitis is the most common form of ocular allergies. The pathogenetic mechanisms are based on an immunoglobulin E (IgE)mediated hypersensitivity reaction. On the other hand, tear osmolarity has been suggested to be an index of ocular surface damage and inflammation. These data were the motive to investigate the levels of tear osmolarity in subjects with acute allergic rhinoconjuctivitis, before and after administration of artificial tears. Patients and Methods: Forty-five subjects with acute allergic rhinoconjuctivitis were randomly divided into three groups, based on the type of artificial tears that they received: Group A (Thera tears), Group B (Wet therapy) and Group C (Tears Naturale free). The eye drops were administered six times a day for 60 days and all subjects underwent grading of subjective symptoms and clinical examination at baseline and at the end of the treatment. Results: The diagnosis of severe eye disease, which was based on ocular surface disease index (OSDI; Allergan, Inc, Irvine, CA, USA) and tear osmolarity values, concerned
\end{abstract}

This article is freely accessible online.

Correspondence to: Dr. Marilita M. Moschos, First Department of Ophthalmology, General Hospital of Athens G. Gennimatas, Medical School, National and Kapodistrian University of Athens; 6, Ikarias Street, Ekali, 14578, Greece. Tel: +30 6944887319, Fax: +30 2104122319, e-mail: moschosmarilita@yahoo.fr

Key Words: Acute allergic rhinoconjuctivitis, artificial tears, tear osmolarity. all patients at baseline. Although the administration of artificial tears significantly ameliorated the symptoms and the ocular variables in all groups, the results were better in the first group. Tear osmolarity was strongly and negatively correlated with tear film breakup time (BUT) and Schirmer I test at 2 months. Contrariwise, symptoms were eliminated, when tear osmolarity was decreased. Conclusion: Acute allergic rhinoconjuctivitis is characterized by tear hyperosmolarity, which can be rehabilitated with the administration of hypotonic artificial tears.

Acute allergic rhinoconjuctivitis is the most common form of ocular allergies, affecting at least $15-20 \%$ of the population. It is divided into seasonal (hay fever), which is released by pollen in the spring and summer, and perennial, which appears throughout the year and is induced by dust mites, animal dander and mold allergens (1, 2). The pathogenetic mechanisms of acute allergic conjunctivitis are based on an immunoglobulin $\mathrm{E}$ ( $\mathrm{IgE}$ )-mediated hypersensitivity reaction, where IgE binds initially to highaffinity receptors on mast cells and basophils and then to the aforementioned allergens. The subsequent activation of mast cells results in the release of inflammatory mediators, including histamine, tryptase, prostaglandins, chemokines and leukotrienes (1). Monocytes, eosinophils and platelets also afford low-affinity $\operatorname{IgE}$ receptors (2). Subjects experience acute episodes of redness, tearing, ocular and nasal itching, sneezing, congestion and rhinorrhoea. Slit lamp examination usually reveals bilateral eyelid edema along with conjunctival swelling, chemosis and papillary reaction $(1,2)$. 
Tear osmolarity has been suggested to be an index of ocular surface damage and inflammation since $1995(3,4)$. Tear hyperosmolarity has been associated not only with dry eye disease (keratoconjunctivitis sicca), exhibiting positive correlation with the severity of the disease, but as well with herpetic keratitis, pterygia, and pseudoexfoliation syndrome $(4,5)$. Ocular medications have been found to modify tear osmolarity, including anti-glaucomatous drugs, which seem to raise the values, and artificial tears, which reduce tear osmolarity (5). Furthermore, the topical administration of $1 \%$ methylprednisolone, in subjects with moderate to severe dry eye syndrome, seems to lower tear osmolarity and cytokine levels (6). The association of tear osmolarity with inflammation has been also highlighted by Türkyilmaz et al., who related tear osmolarity values with the degree of early rheumatoid arthritis (7).

The inflammatory background of acute allergic rhinoconjuctivitis along with the records of tears hyperosmolarity in inflammatory diseases was the motive to investigate tear osmolarity in patients with acute allergic rhinoconjuctivitis. The aim of this study was to estimate the levels of tear osmolarity in subjects with acute allergic rhinoconjuctivitis, assessing in parallel the effect of three different types of artificial tears on tear osmolarity values.

\section{Patients and Methods}

Patient recruitment. Forty-five subjects with acute allergic rhinoconjuctivitis were recruited in this study. An experienced allergist referred to us patients with ocular symptoms, who had never been treated by an ophthalmologist. Although acute allergic rhinoconjuctivitis has already been defined (8), we mention that we diagnosed the disease in people suffering from the following symptoms: nasal obstruction, watery nasal discharge, sneezing and itching, and conjunctival symptoms, including itching, injection and tearing. The inclusion criteria also involved the follow indexes: tear film breakup time (BUT) less than 5 seconds, Schirmer I test less than $5 \mathrm{~mm}$, and positive corneal and conjunctival staining. Past medical history, including medications, medical conditions, ocular history, within the last 3 months was obtained.

The exclusion criteria comprised any evidence of acute or chronic infection, any inflammatory or allergic condition of the cornea and conjunctiva other than acute allergic rhinoconjuctivitis, incomplete lid closure, entropion, ectropion, nasolacrimal drainage obstruction, placement of punctal plugs or punctal cauterisation, history of ocular surgery within the past year, use of contact lenses during the previous month and use of other topical ocular medications or artificial tears within 2 hours of checking tear osmolarity. Moreover, the history of known collagen vascular disease, such as secondary Sjögren syndrome or positive serology of autoantibodies, was regarded as exclusion criteria. Subjects suffering from systemic diseases, such as diabetes mellitus and thyroid diseases, or receiving treatment, including antidepressants, diuretics, corticosteroids or immunomodulators, which could impact tear secretion or the ocular surface, were ruled out by the medical history.
Study design. This study was designed as a prospective study. All subjects included were treated for a run-in period of 7 days with one eye drop of saline six times a day. At the end of this period (time 0 of the study) the subjects were randomly divided into three groups and assigned to a treatment by personnel not involved with the subjects' examination. The number of the groups was determined according to the type of artificial tears that each patient received: Group A (Thera tears), Group B (Wet therapy) and Group C (Tears Naturale free). The eye drops were administered six times a day for 60 days and the subjects enrolled in the study underwent grading of subjective symptoms and clinical examination at baseline (time 0) and at the end of the treatment (after 60 days).

The parameters evaluated at baseline and at each follow up visit included the ocular surface disease index (OSDI; Allergan, Inc, Irvine, CA, USA), BUT, tear osmolarity test, Schirmer test I, fluorescein corneal and conjunctival staining. Tear osmolarity was measured first and Schirmer test was performed last, in order to minimize the effect of other examinations. The subjects were allowed to know the brand name of the eye drops they were using. They were asked to bring, at all visits, the used boxes of eye drops in order to control the compliance with the study protocol.

The study was conducted in the General Hospital of Athens G. Gennimatas in accordance to the tenets of the Declaration of Helsinki. The protocol used was approved by the ethics committee of the University Hospital. All patients agreed to participate in the study and gave written informed consent.

Study material. We used the following types of artificial tears: Thera tears (Akorn, Inc., Lake Forest, Illinois, USA), Wet therapy (Vita Research, Rome, Italy) and Tears Naturale free (Alcon, Inc., Fort Worth, Texas, USA). The characteristics of all drops are depicted in Table I. All artificial tears were preservative-free.

Symptoms. Symptoms of dry eye syndrome, such as burning, foreign body sensation, dryness, mucous discharge, itching and photophobia, were assessed with the ocular surface disease index (OSDI; Allergan, Inc, Irvine, CA, USA). The OSDI consists of 12 questions on symptoms within the past week and yields scores ranging from 0 (least severe) to 100 (most severe). A score of 12 is typically used as a cutoff for normal, 13-22 for mild dry eye, 23-32 for moderate dry eye, and $\geq 33$ for severe dry eye $(1,2,9)$.

Tear osmolarity. Tear osmolarity was measured in each eye with the TearLab $^{\circledR}$ Osmolarity System (TearLab Corporation, San Diego, CA, USA). This new osmometer is a disposable 'lab-on-a-chip' system that requires $<50 \mathrm{nl}$ of tear fluid for analysis (3). A desktop instrument converts the electrical signals generated from the laboratory card into a quantitative measurement and displays the value on a screen. The system was stored in a temperature and humidity controlled environment, and these values were logged prior to each measurement of tear osmolarity. The system was calibrated at the beginning of each study day according to the manufacturer's instructions; the test cards used for each tear osmolarity measurement were from the same lot numbers as the test cards used to calibrate the machine each day.

Subjects were instructed not to rub their eyes for at least 10 minutes. Tear samples (50 $\mathrm{nl}$ each sample) were collected atraumatically from the lateral tear meniscus (near the lateral canthus) of the right eye and then the left eye. The tip of the TearLab device (Pen 1) was placed gently with care being taken not to induce reflex tearing. Normal tear osmolarity is equivalent to 
Table I. The characteristics of artificial tears used in the study.

\begin{tabular}{|c|c|c|c|}
\hline & Substances & Excipients & Osmolarity \\
\hline Thera tears (preservative free) & $\begin{array}{c}\text { Sodium carboxymethyl } \\
\text { Cellulose } 0.25 \%\end{array}$ & $\begin{array}{c}\text { Borate buffers } \\
\text { Calcium chloride } \\
\text { Magnesium chloride } \\
\text { Potassium chloride } \\
\text { Purified water } \\
\text { Sodium bicarbonate } \\
\text { Sodium chloride } \\
\text { Sodium phosphate }\end{array}$ & Hypotonic \\
\hline Wet therapy (no preservatives) & $\begin{array}{c}\text { Sodium hyaluronate (MW=14X 105) } 0.15 \% \\
\text { Polyethylene glycol (PEG 400) } 0.20 \%\end{array}$ & $\begin{array}{c}\text { Purified water } \\
\text { Boric acid } \\
\mathrm{NaCl}, \mathrm{KCl}, \mathrm{CaCl}_{2}, \mathrm{MgCl}_{2}\end{array}$ & Isotonic \\
\hline Tears Naturale free & $\begin{array}{l}\text { Hypromellose } 2910(3 \mathrm{mg} / \mathrm{ml}) \\
\text { Dextran } 70(1 \mathrm{mg} / \mathrm{ml})\end{array}$ & $\begin{array}{c}\text { Hydrochloric acid } \\
\text { Sodium borate decahydrate } \\
\text { Sodium chloride } \\
\text { Sodium hydroxide } \\
\text { Polidronium chloride } \\
\text { Potassium chloride } \\
\text { Purified water }\end{array}$ & Isotonic \\
\hline
\end{tabular}

normal blood osmolarity, which ranges from 280-295 mOsm/L. A tear osmolarity value of $305 \mathrm{mOsm} / \mathrm{l}$ was used as the cutoff for mild dry eye and $316 \mathrm{mOsm} / 1$ was used as the cut-off for more severe dry eye disease $(3,4)$. All measurements were performed by the same investigator. Since inter-eye variability has been found to be associated with an increased severity of DED, the measurement from the eye with the higher osmolarity was used for data analysis.

BUT and Schirmer test. Fluorescein strip was wet with a dropvolume of non-preserved saline solution and the strip was touched to the inferior palpebral conjunctiva. Subjects were asked to blink several times. The investigator monitored the integrity of the tear film with a slit lamp and measured up to the time until one or more dry spots appeared in the precorneal tear film from the last blink.

A 5-minute Schirmer I test without topical anesthesia was performed to evaluate both basal tear secretion and reflex tearing. A Schirmer test I value of less than $10 \mathrm{~mm}$ in 5 minutes was considered abnormal (5).

Corneal staining. The degree of staining was measured for each of the five regions of the cornea: central, superior, temporal, nasal, and inferior. The degree of staining was evaluated using the National Eye Institute (NEI) method, for each of the five regions of the cornea (central, inferior, nasal, superior, and temporal) as follow: 1) grade 0 (normal); no staining, 2) grade 1 (mild); superficial stippling and micropunctate staining, 3) grade 2 (moderate); macropunctate staining with some coalescent areas; and 4) grade 3 (severe); numerous coalescent macropunctate areas and/or patches. Each of the five regions was graded on a scale from 0 to 3 . The scores of the five areas were added to obtain a total score for each eye.

Conjunctival staining. The degree of staining was separately assessed for the three portions of the temporal conjunctiva and the three portions of the nasal conjunctiva on a scale from 0 to 3 . The scores for each of the six areas were added to obtain a total score for each eye.
Statistical analysis. The statistical program IBM SPSS Statistics 22.0 was used for the data analysis. Descriptive analysis was carried out for age, OSDI scores, tear osmolarity, BUT and Schirmer I test values, as well as corneal and conjunctival staining scores. Non-parametric analysis Kolmogorov-Smirnov was used to check the normal distribution of the variables. The paired twotailed $t$-test was utilized to calculate the differences in means of the measured variables between baseline and after the administration of artificial tears within each group. If the data failed the normality test, the non-parametric Wilcoxon matched-pairs signed-rank test was used. Independent samples Kruskal-Wallis tests were applied to identify the possible differences in means of the measured variables between the three groups (Mann-Whitney test was used when there was no indication of normal distribution either after Kolmogorov-Smirnov analysis or after Levene's test for e3quality of variances). Correlation technique, based on Spearman's coefficient, was performed to determine the relation among ocular indexes. A $p$-value less than 0.05 was considered to indicate statistical significance.

\section{Results}

Demographics. Forty-five subjects with acute allergic rhinoconjuctivitis were classified into three groups according to the type of artificial tears, which we prescribed to them. The number of males were $8(0.53 \%), 9(0.6 \%)$ and 10 $(0.67 \%)$ in groups $\mathrm{A}, \mathrm{B}$ and $\mathrm{C}$, respectively. The relative numbers for females were $7(0.47 \%), 6(0.4 \%)$ and 5 $(0.33 \%)$, respectively. The mean distribution of age within each group is presented in Table II. No statistically significant differences in the distributions of gender (Pearson chi-square test, $p=0.757$ ) and age (one-way Anova, $p=0.853$ ) were observed among three groups. 
Table II. Descriptive data of measured variables and the comparisons tests.

\begin{tabular}{|c|c|c|c|c|}
\hline Mean (SD) & Group A & Group B & Group C & $p$-Value \\
\hline Age (years) & $41(5.8)$ & $41(5.3)$ & $40(5.4)$ & - \\
\hline OSDI1(at baseline) & $64.0(7.8)$ & $66.0(5.1)$ & $66.3(5.6)$ & 0.891 \\
\hline OSDI2 (at 2 months) & $19.9(4.2)$ & $36.1(2.0)$ & $32.8(4.1)$ & $<0.001$ \\
\hline Tear osmolarity (mOsm/L) (at baseline) & $318.1(8.2)$ & $324.3(5.4)$ & $323.5(4.4)$ & 0.059 \\
\hline Tear osmolarity (mOsm/L) (at 2monts) & $292.1(5.4)$ & $313.1(5.9)$ & $313.3(4.3)$ & $<0.001$ \\
\hline BUT1 (sec) (at baseline) & $2.7(0.9)$ & $2.7(1.2)$ & $2.5(1.1)$ & 0.751 \\
\hline BUT2 (sec) (at 2 months) & $7.7(1.0)$ & $4.7(1.2)$ & $5.3(0.7)$ & $<0.001$ \\
\hline Schirmer test I (mm) (at baseline) & $2.9(0.9)$ & $2.9(0.8)$ & $2.8(0.7)$ & 0.810 \\
\hline Schirmer test I (mm) (at 2months) & $8.1(2.4)$ & $6.1(1.1)$ & $5.3(1.0)$ & $<0.001$ \\
\hline Corneal staining score (at baseline) & $1.5(0.5)$ & $1.4(0.5)$ & $1.4(0.5)$ & 0.770 \\
\hline Corneal staining score (at 2 months) & $0.5(0.5)$ & $1.0(0.4)$ & $0.9(0.4)$ & 0.005 \\
\hline Conjunctival staining score (at baseline) & $1.4(0.5)$ & $1.5(0.5)$ & $1.5(0.5)$ & 0.770 \\
\hline Conjunctival staining score (at 2 months) & $0.5(0.5)$ & $1.1(0.3)$ & $0.9(0.4)$ & 0.001 \\
\hline
\end{tabular}

${ }^{*} p$-Value was extracted using Independent samples Kruskal-Wallis tests.

Ocular surface indexes. The mean values of OSDI, tear osmolarity, BUT, Schirmer I test, as well as corneal and conjunctival staining scores are presented in Table II. The mean values of OSDI, tear osmolarity, BUT, Schirmer I test, corneal and conjunctival staining scores exhibited no statistical significant differences at baseline among the three groups. Contrariwise, significant differences in the means of all variables were detected 2 months after the administration of the artificial tears. Actually, tear osmolarity was significantly reduced in all groups, but only in Group A the levels restored to normal (Table II). Furthermore, BUT, Schirmer I test, as well as corneal and conjunctival staining scores were all decreased in three groups, but the values were better in group A (Table II). The alterations of tear osmolarity, BUT, Schirmer I test, corneal and conjunctival staining scores were all significant between baseline and 2 months after treatment (Table II). The statistical significance of the differences observed in ocular indexes and symptoms of dry eye disease after the administration of artificial tears in all 3 groups are demonstrated in Table III.

Correlations were performed to determine the relation among ocular indexes recorded in three groups. Tear osmolarity recorded in all groups at 2 months treatment, was positively and significantly correlated with tear osmolarity at baseline (Spearman's coefficient $=0.38, p=0.010$ ), with OSDI2 (Spearman's coefficient $=0.64, \quad p<0.001$ ), with corneal (Spearman's coefficient $=0.37, p=0.013$ ), and conjunctival staining at 2 months (Spearman's coefficient $=0.45, p=0.002$ ). On the other hand, it was negatively and significantly correlated with BUT2 (Spearman's coefficient $=-0.78$, $p<0.001$ ) and Schirmer I test at 2 months (Spearman's coefficient $=-0.60, p<0.001)$.

BUT2 was positively and significantly correlated with BUT1 (Spearman's coefficient $=0.45, p=0.002$ ) and Schirmer I test at 2 months (Spearman's coefficient $=-0.58, p<0.001$ ) among participants. BUT2 recorded in all groups was negatively and significantly correlated with OSDI2 (Spearman's coefficient=0.66, $p<0.001$ ), corneal (Spearman's coefficient $=0.34$, $p=0.023$ ), and conjunctival staining at 2 months (Spearman's coefficient $=0.52, p<0.001)$. A significant negative correlation was also detected in three groups between OSDI 2 and Schirmer I test at 2 months (Spearman's coefficient $=-0.57, p<0.001$ ), while the same score was positively correlated with corneal (Spearman's coefficient $=0.49, p=0.001$ ), and conjunctival staining at 2 months (Spearman's coefficient $=0.42, p=0.004$ ).

Moreover, a significant and positive correlation was detected between corneal staining at 2 months and both with corneal staining at baseline (Spearman's coefficient $=0.45$, $p=0.002$ ), as well as with conjunctival staining at 2 months (Spearman's coefficient $=0.43, p=0.003$ ) among participants. Similarly, the scores of conjunctival staining recorded in three groups at baseline and at 2 months were positively and significantly correlated (Spearman's coefficient $=0.31$, $p=0.037)$. Schirmer I tests at baseline and after 2 months were significantly and positively correlated (Spearman's coefficient $=0.30, p=0.044$ ) among participants.

\section{Discussion}

In our study we noted that all subjects suffered from severe eye disease at baseline, based on OSDI scores and tear osmolarity values. Moreover, the mean values of OSDI, tear osmolarity, BUT, Schirmer I test, corneal and conjunctival staining scores exhibited no statistical significant differences at baseline among the three groups. Although the administration of artificial tears ameliorated significantly the symptoms (OSDI scores), the tear film osmolarity and the rest ocular variables in all groups, the results were better in the first group. Specifically, the use of hypotonic eye drops altered 
Table III. Statistical significance of the differences observed in ocular indexes and symptoms after the administration of artificial tears ( $p$ values extracted by Wilcoxon matched-pairs signed-rank tests). Wilcoxon matched-pairs signed-rank tests

\begin{tabular}{lccc}
\hline & Group A & Group B & Group C \\
\hline Tear osmolarity (mOsm/L) & 0.001 & 0.001 & 0.001 \\
BUT (sec) & 0.001 & $<0.001$ & 0.001 \\
Schirmer test I (mm) & 0.001 & 0.001 & 0.001 \\
Corneal staining score & $<0.001$ & 0.008 & 0.005 \\
Conjunctival staining score & 0.001 & 0.008 & 0.003 \\
OSDI & 0.001 & 0.001 & 0.001 \\
\hline
\end{tabular}

BUT: Tear film breakup time; OSDI: ocular surface disease index.

the OSDI severity into mild and rehabilitated tear osmolarity to normal. Subjects who used the other types of artificial tears exhibited also severe symptoms after 2 months administration, while the increase of tear osmolarity was eliminated in mild stages. Furthermore, we noted that tear osmolarity was correlated strongly and negatively with BUT2 and Schirmer I test at 2 months, being reduced when BUT2 and Schirmer test were raised. Contrariwise, symptoms (OSDI2) were eliminated, when tear osmolarity was decreased.

Tears hyperosmolarity observed in subjects with keratoconjunctivitis sicca ranges between $311-425 \mathrm{mOsm} / \mathrm{L}$. It is estimated to be a sensitive (sensitivity 90-95\%) and specific (specificity 94-95\%) index for the diagnosis of dry eye syndrome $(10,11)$. Lemp et al. noted that tear osmolarity exhibited $73 \%$ sensitivity and $92 \%$ specificity for the diagnosis of dry eye syndrome (12). Recent study revealed no significant difference in means of tear osmolarity, measured by TearLab Osmolarity System, between healthy individuals and subjects with dry eye syndrome. Furthermore, no alteration of tear osmolarity was detected after treatment with autologous serum eye drops (13). On the other hand, topical administration of $1 \%$ methylprednisolone four times per day seemed to decrease tear osmolarity and cytokine levels, along with the improvement of BUT, corneal and conjunctival staining scores, at 4th and 8th weeks (6). Suzuki et al. assessed tear osmolarity, BUT, Schirmer I test, as well as corneal and conjunctival staining scores in subjects with Dry Eye Syndrome and the measured values were as follow: $309.7 \pm 22.3 \mathrm{mOsm} / 1,6.1 \pm 3.3 \mathrm{sec}, 10.6 \pm 6.6 \mathrm{~mm}, 4.9 \pm 3.6$ and $3.5 \pm 3.0$, respectively. Moreover, they correlated tear osmolarity (measured with a tear osmometer) negatively with Schirmer I test and positively with dry eye severity grade (14).

Bunya et al. measured tear osmolarity with TearLab Osmolarity System in 49 subjects with Sjogren's syndrome, who had a mean value of $314.5 \mathrm{mOsm} / \mathrm{L}$. Tear osmolarity was significantly and negatively correlated both with Schirmer I test and OSDI. The later finding was attributed to decreased corneal sensitivity (15). Aragona et al. assessed dry eye disease in subjects with Sjogren's syndrome with corneal fluorescein and conjunctival rose Bengal vital staining, BUT measurements, Schirmer's I test and conjunctival impression cytology. They revealed that hypotonic sodium hyaluronate eye drops can be more beneficial than isotonic ones in treating the disease (16). Ocular mucous membrane pemphigoid, an autoimmune disease, has been also associated with tear hyperosmolarity (mean value 322.90 \pm 33.39 $\mathrm{mOsm} / \mathrm{L}$ ) by Miserocchi et al., who positively correlated it with BUT (mean value $6.60 \pm 3.13 \mathrm{sec}$ ) in a sample of 40 subjects. They observed no significant correlation of tear osmolarity with Schirmer test or with the OSDI score (17).

Tear osmolarity (measured by TearLab Osmolarity System) has been found to be also increased in parallel with the severity of ocular graft-vs.-host disease, exhibiting high sensitivity ( $98.4 \%$ sensitivity), but less specificity ( $60.7 \%$ specificity). The same study group supported that corneal staining score and OSDI score gradually raised along with the severity of the disease in 63 subjects with different hematologic diseases (18). Türkyilmaz et al. observed that tear osmolarity was positively and significantly correlated with the activity of early rheumatoid arthritis. The observed mean values of tear osmolarity, BUT and Schirmer I test were $314.2 \pm 11.4 \mathrm{mOsm} / \mathrm{L}, 6.9 \pm 3.9 \mathrm{sec}$ and $9.4 \pm 4.2 \mathrm{~mm}$, respectively (7). Higher tear osmolarity (mean value $320.40 \pm 21.80 \mathrm{mOsm} / \mathrm{l}$ ) has been also noted in diabetic subjects, where it was related to the duration of Diabetes Mellitus (19). The raised proptosis and lid fissure width appeared to be the reasons for tear hyperosmolarity (mean value $290.80 \pm 13.58 \mathrm{mOsm}$ ), as assessed by an auto-osmometer (OM6030 AUTO STAT; Daiichi, Kyoto, Japan), in 21 subjects with thyroid ophthalmopathy (20).

Aslan et al. investigated the impact of osmoprotective artificial tears on ocular surface disturbances observed in contact lens wearers. They found that tear osmolarity (measured by TearLab osmolarity system) was elevated within the first hours after the application of contact lenses in subjects who had never wore contact lenses previously. The subsequent installation of osmoprotective eye drops (Optive and Refresh, Allergan) appeared to prevent tear hyperosmolarity (21). Ozsutcu et al. estimated that eyes with pterygium had higher tear osmolarity levels (mean $307 \mathrm{mOsm} / 1$ ), corneal staining (mean $1.2 \pm 1.1$ ), and conjunctival redness (mean $0.9 \pm 0.9$ scores), but lower BUT (mean $10.3 \pm 3.4 \mathrm{sec}$ ) and Schirmer I test (mean $14.8 \pm 9.2 \mathrm{~mm}$ ) values than control eyes (22). A recent study investigated tear osmolarity, using TearLab system, in subjects with glaucoma who received at least one anti-glaucomatous medication (50 subjects) over six months or underwent trabeculectomy (31 subjects) over six months without using any medication. Tear osmolarity was higher in both groups (means $307.0 \pm 9.3$ and $307.4 \pm 11.6 \mathrm{mOsm} / 1$, respectively) compared to healthy individuals (mean $301.4 \pm 7.7 \mathrm{mOsm} / \mathrm{l}$ ). 
However, no statistically significant differences neither in tear osmolarity nor in BUT and Schirmer's test scores were detected among the three groups (23).

\section{Conclusion}

This is the first study to investigate tear osmolarity in subjects with acute allergic rhinoconjuctivitis. Even if the sample size was relatively small, but comparable to the sizes used in previous studies assessing tear osmolarity, we concluded that acute allergic rhinoconjuctivitis is an additional cause of tear hyperosmolarity. The inflammatory background of the disease is probably related to these observations. Furthermore, we revealed that the administration of hypotonic artificial tears can prevent from ocular surface damage, rehabilitating tear osmolarity.

\section{References}

1 La Rosa M, Lionetti E, Reibaldi M, Russo A, Longo A, Leonardi $\mathrm{S}$, Tomarchio S, Avitabile $\mathrm{T}$ and Reibaldi A: Allergic conjunctivitis: a comprehensive review of the literature, Ital J Pediatr 39: 18, 2013.

2 Andersson M, Greiff L and Svensson C: Allergic rhinoconjunctivitis: the role of histamine. Mediators Inflamm 3: 171-175, 1994.

3 Tomlinson A, Khanal S, Ramaesh K, Diaper C and McFadyen A: Tear film osmolarity: determination of a referent for dry eye diagnosis. Invest Ophthalmol Vis Sci 47: 4309-4315, 2006.

4 DEWS Definition and Classification. The definition and classification of dry eye disease: Report of the Definition and Classification Subcommittee of the International Dry Eye Workshop. Ocul Surf 5: 75-92, 2007.

5 Potvin R, Makari S and Rapuano CJ: Tear film osmolarity and dry eye disease: a review of the literature. Clin Ophthalmol 9: 2039-2047, 2015.

6 Lee JH, Min K, Kim SK, Kim EK and Kim TI: Inflammatory cytokine and osmolarity changes in the tears of dry eye subjects treated with topical $1 \%$ methylprednisolone. Yonsei Med J 55: 203-208, 2014.

7 Türkyilmaz K, Küçükali Türkyilmaz A, Kurt A, Kurt EE, Sevim MŞ and Oner V: Investigation of tear osmolarity in early rheumatoid arthritis: relation to disease activity. Can J Ophthalmol 48: 235-239, 2013.

8 Dhami S, Nurmatov U, Roberts G, Pfaar O, Muraro A, Ansotegui IJ, Calderon M, Cingi C, Demoly P, Durham S, van Wijk RG, Halken S, Hamelmann E, Hellings P, Jacobsen L, Knol E, Linnemann DL, Lin S, Maggina V, Oude-Elberink H, Pajno G,Panwankar R, Pastorello E, Pitsios C, Rotiroti G, Timmermans F, Tsilochristou O, Varga EM, Wilkinson J, Williams A, Worm M, Zhang L and Sheikh A: Allergen immunotherapy for allergic rhinoconjunctivitis: protocol for a systematic review. Clin Transl Allergy 22: 6-12, 2016.

9 Semeraro F, Forbice E, Nascimbeni G, Taglietti M, Romano V Guerra G, and Costagliola C: Effect of autologous serum eye drops in patients with sjögren syndrome-related dry eye: clinical and in vivo confocal microscopy evaluation of the ocular surface. In Vivo 30: 931-938, 2016.
10 Lucca JA, Nunez JN and Farris RL: A comparison of diagnostic tests for keratoconjunctivitis sicca: lactoplate, Schirmer, and tear osmolarity. CLAO J 16: 109-112, 1990.

11 Gilbard JP, Farris RL and Santamaria J 2nd: Osmolarity of tear microvolumes in keratoconjunctivitis sicca. Arch Ophthalmol 96: 677-681, 1978.

12 Lemp MA, Bron AJ, Baudouin C, Benítez Del Castillo JM, Geffen D, Tauber J, Foulks GN, Pepose JS and Sullivan BD: Tear osmolarity in the diagnosis and management of dry eye disease. Am J Ophthalmol 51: 792-798, 2011.

13 Mahelková G, Veselá V, Seidler Štangová P, Židlická A, Dotřelová D, Fales I, Skalická P and Jirsová K: Tear osmolarity in subjects with severe dry eye syndrome before and after autologous serum treatment: a comparison with tear osmolarity in healthy volunteers. Cesk Slov Oftalmol 71: 184-188, 2015.

14 Suzuki M, Massingale ML, Ye F, Godbold J, Elfassy T, Vallabhajosyula $\mathrm{M}$ and Asbell PA: Tear osmolarity as a biomarker for dry eye disease severity. Invest Ophthalmol Vis Sci 51: 4557-4561, 2010.

15 Bunya VY, Langelier N, Chen S, Pistilli M, Vivino FB and Massaro-Giordano G: Tear osmolarity in Sjögren syndrome. Cornea 32: 922-927, 2013.

16 Aragona P, Di Stefano G, Ferreri F, Spinella R and Stilo A: Sodium hyaluronate eye drops of different osmolarity for the treatment of dry eye in Sjögren's syndrome subjects. Br J Ophthalmol 86: 879-884, 2002.

17 Miserocchi E, Iuliano L, Berchicci L, Bandello F and Modorati G: Tear film osmolarity in ocular mucous membrane pemphigoid. Cornea 33: 668-672, 2014.

$18 \mathrm{Na}$ KS, Yoo YS, Hwang KY, Mok JW and Joo CK: Tear osmolarity and ocular surface parameters as diagnostic markers of ocular graft-versus-host disease. Am J Ophthalmol 160: 143$149,2015$.

19 Sağdık HM, Ugurbas SH, Can M, Tetikoğlu M, Ugurbas E, Uğurbaş SC, Alpay A and Uçar F: Tear film osmolarity in subjects with diabetes mellitus. Ophthalmic Res 50: 1-5, 2013.

20 Iskeleli G, Karakoc Y and Abdula A: Tear film osmolarity in subjects with thyroid ophthalmopathy. Jpn J Ophthalmol 52: 323-326, 2008.

21 Aslan Bayhan S, Bayhan HA, Muhafız E, Bekdemir Ş and Gürdal C: Effects of osmoprotective eye drops on tear osmolarity in contact lens wearers. Can J Ophthalmol 50: 283-289, 2015.

22 Ozsutcu M, Arslan B, Erdur SK, Gulkilik G, Kocabora SM and Muftuoglu O: Tear osmolarity and tear film parameters in subjects with unilateral pterygium. Cornea 33: 1174-1178, 2014.

23 Lee SY, Wong TT, Chua J, Boo C, Soh YF and Tong L: Effect of chronic antiglaucoma medications and trabeculectomy on tear osmolarity. Eye (Lond) 27: 1142-1150, 2013 